\title{
Dissecção isolada do tronco celíaco: relato de caso
}

\author{
Isolated dissection of the celiac artery: a case report
}

José Manoel da Silva Silvestre', Wander Eduardo Sardinha², Marcelo Piazzalunga³, Benedito Fernandes ${ }^{4}$, Fernando Motta Guilherme da Silva Silvestre ${ }^{6}$

\section{Resumo}

A dissecção espontânea isolada do tronco celíaco sem a dissecção concomitante da aorta é uma condição rara, pouco descrita na literatura. O objetivo do presente trabalho é descrever um caso dessa entidade clínica em um paciente masculino, 74 anos, assintomático, cujo diagnóstico foi feito por ultrassonografia e confirmado com angiotomografia computadorizada. O paciente tem sido acompanhado com sucesso mediante observação clínica por um período de 25 meses.

Palavras-chave: Artéria celíaca, dissecção, ultrassonografia.

\begin{abstract}
The isolated spontaneous dissection of the celiac artery without the concomitant dissection of the aorta is a rare condition seldomly described in the literature. The objective of the present study is to describe a case of this clinical entity in a 74-year-old, male patient, who was asymptomatic, and whose diagnosis was established by means of ultrasound and confirmed using computed angiotomography. The patient has been successfully followed up by means of clinical management for a period of 25 months.
\end{abstract}

Keywords: Celiac artery, dissection, ultrasonography.

Introdução

A dissecção espontânea de uma artéria visceral, principalmente quando não associada a uma dissecção aórtica, é considerada uma entidade rara. Dentre as dissecções das artérias viscerais, as que comprometem a artéria mesentérica superior são as mais comuns, sendo as do tronco celíaco e da artéria mesentérica inferior consideradas muito infrequentes ${ }^{1-3}$.

Nosso objetivo é descrever um caso de dissecção espontânea de tronco celíaco em paciente assintomático, onde se optou apenas por seguimento clínico, sendo acompanhado por um período de 25 meses com evolução satisfatória.

\section{Relato do caso}

Paciente de 74 anos, sexo masculino, foi encaminhado para o atendimento por cirurgião vascular por ter tido como achado de exame ultrassonográfico de rotina de abdome uma imagem compatível com dissecção de tronco celíaco. O paciente negava quadro de dor abdominal nos últimos meses. Entre seus antecedentes, referia apenas hiperlipidemia em uso de estatina e tabagismo abandonado 40 anos atrás.

O exame físico não revelava nenhuma alteração significativa, sendo o abdome indolor, flácido e sem tumorações palpáveis. Os pulsos periféricos estavam presentes e normais.

\footnotetext{
Trabalho apresentado no Congresso Internacional de Cirurgia Endovascular, São Paulo, em abril de 2009.

'Doutor. Professor associado, Disciplina de Angiologia e Cirurgia Vascular, Universidade Estadual de Londrina (UEL), Londrina PR.

${ }^{2}$ Doutor. Professor associado, Disciplina de Angiologia e Cirurgia Vascular, UEL, Londrina, PR.

${ }^{3}$ Médico responsável pela Angiotomografia da Ultramed - Diagnóstico por Imagem, Londrina, PR.

${ }^{4}$ Médico. Especialista em Ultrassonografia. Ultramed - Diagnóstico por Imagem, Londrina, PR.

${ }^{5}$ Médico residente de Angiorradiologia e Cirurgia Endovascular, UEL, Londrina, PR.

${ }^{6}$ Estudante de Medicina, Universidade Estadual do Oeste do Paraná (UNIOESTE), Cascavel, PR.

Não foram declarados conflitos de interesse associados à publicação deste artigo.

Artigo submetido em 27.10.09, aceito em 19.11.09.

J Vasc Bras. 2010;9(1):
} 
A ultrassonografia com Dopplerfluxometria a cores das artérias viscerais demonstrou o tronco celíaco com moderada dilatação fusiforme (Figura 1) que se iniciava 8 mm após sua emergência, com extensão de $19 \mathrm{~mm}$, diâmetros máximos de 18 x $18 \mathrm{~mm}$ e imagens sugestivas de lâmina de dissecção (Figura 2). Os diâmetros no segmento proximal da artéria eram de $8 \times 8 \mathrm{~mm}$. As artérias mesentéricas, superior e inferior, apresentavam-se com diâmetros e fluxos normais.

Solicitou-se angiotomografia computadorizada de aorta abdominal com técnica helidoical, multislice e reconstrução tridimensional. Este exame demonstrou achados semelhantes aos da ultrassonografia, com diâmetros transversos

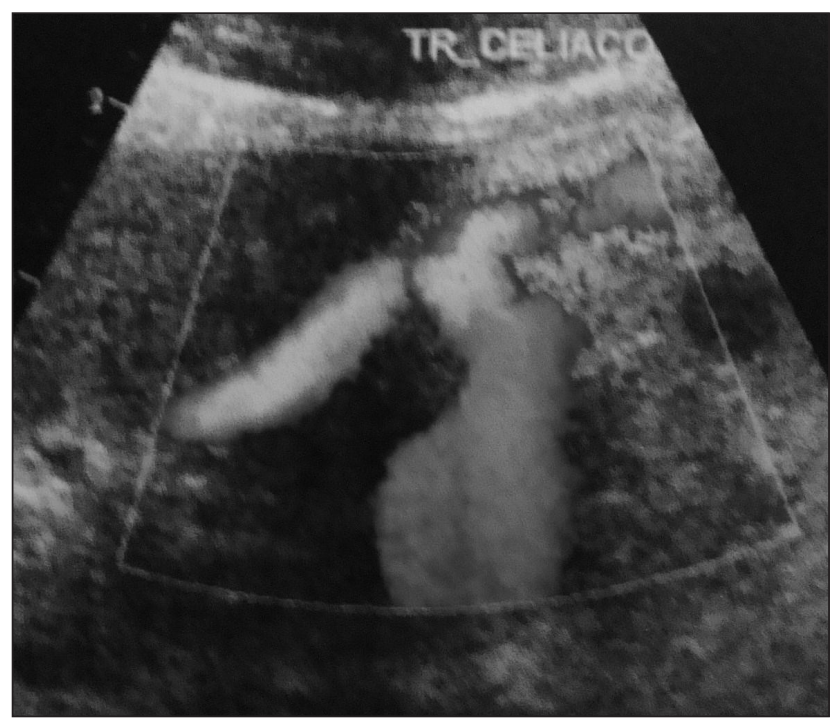

Figura 1 - Ultrassonografia com Dopplerfluxometria a cores demonstrando dilatação do tronco celíaco

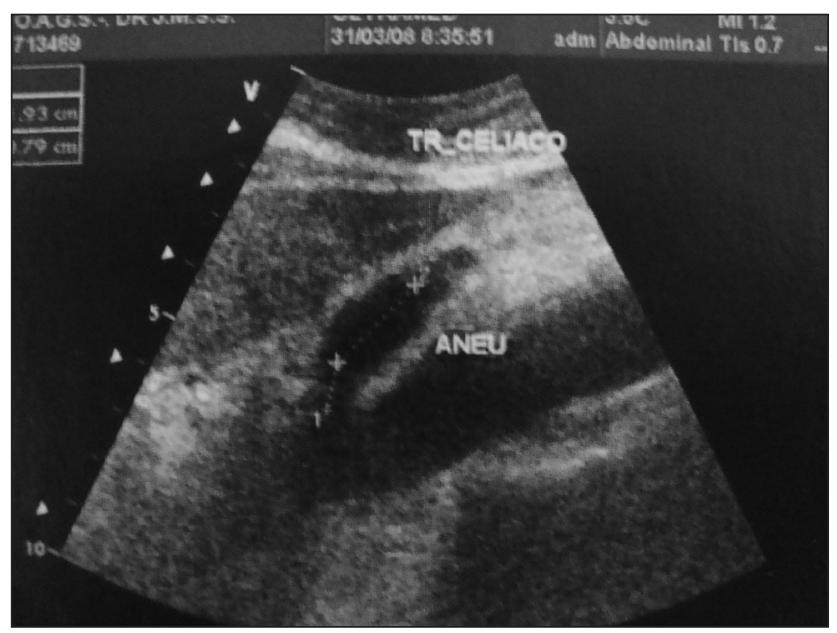

Figura 2 - Ultrassom em modo B: dilatação do tronco celíaco próximo da sua origem na aorta do tronco celíaco de 17,3 x 15,9 mm e lâmina de dissecção no interior do aneurisma (Figuras 3 e 4). A aorta abdominal apresentava placas ateromatosas calcificadas e não calcificadas, sem estenoses significativas, com diâmetros normais e sem imagens de dissecção. As artérias mesentéricas superior e inferior, assim como as artérias renais, apresentavamse normais (Figura 5).

Como o paciente se apresentava assintomático, optou-se apenas pelo acompanhamento seriado, sem o emprego de nenhuma medicação. O exame ultrassonográfico tem sido realizado a cada 6 meses e não mostra alterações, não tendo havido aumento do diâmetro do aneurisma, extensão da área de dissecção ou oclusão dos ramos do tronco celíaco.

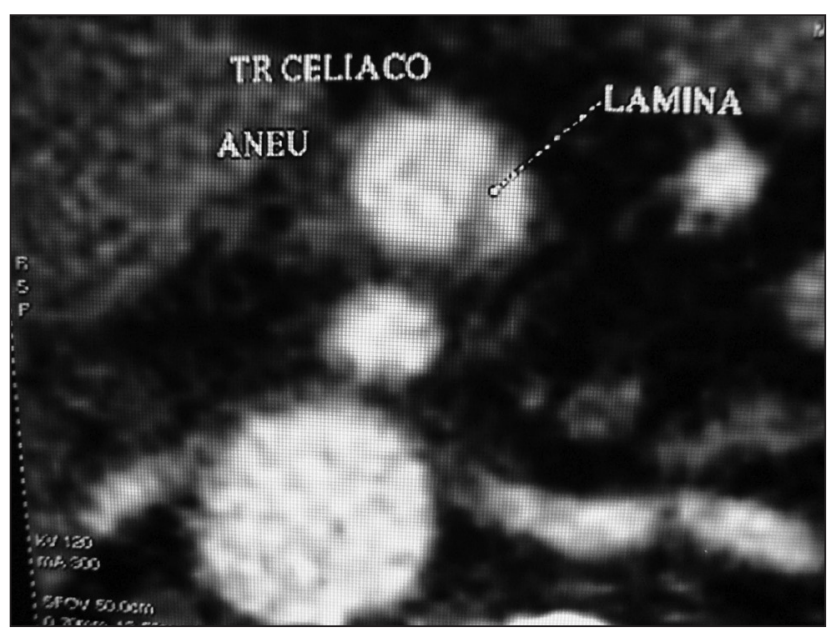

Figura 3 - Angiotomografia demonstrando lâmina de dissecção

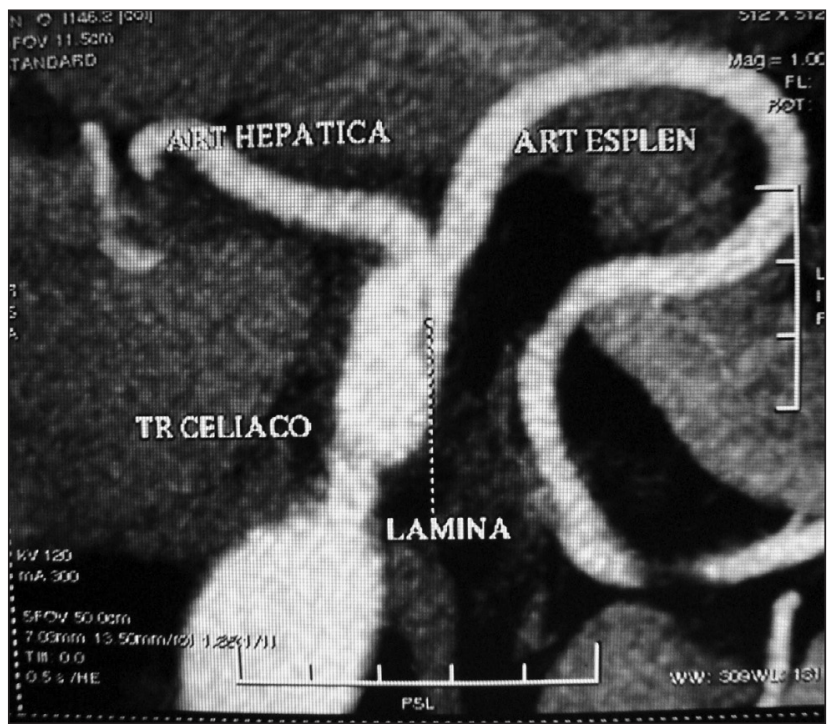

Figura 4 - Lâmina de dissecção 


\section{Discussão}

A dissecção arterial pode ser definida como uma clivagem da parede arterial entre duas camadas elásticas e formação de hematoma local².

A dissecção isolada de uma artéria não associada a uma dissecção aórtica tem sido relatada nas artérias renais e carótidas, porém raramente nas artérias viscerais ${ }^{1,3-8}$. As dissecções extra-aórticas em ordem de frequência decrescente incluem a artéria renal, artéria coronária, artéria cerebral, artéria carótida, artéria vertebral e artérias viscerais ${ }^{9}$. Provavelmente essas dissecções não foram diagnosticadas no passado antes da era dos métodos complementares de imagem.

Dentre as dissecções das artérias viscerais, as da artéria mesentérica superior são as mais frequentes ${ }^{10}$, sendo raras as da artéria mesentérica inferior e as do tronco celíaco e seus ramos. Em uma série de 19 pacientes com dissecção dessas artérias, Takayama et al. ${ }^{10}$ encontraram comprometimento da artéria mesentérica superior em $57,9 \%$, do tronco celíaco em $36 \%$, da artéria hepática isolada em $10,5 \%$ e somente em 5,3\% na artéria esplênica.

Entre os fatores etiológicos, a arteriosclerose é a que tem sido mais frequentemente encontrada. São relatados ainda a degeneração cística da média, trauma, infecções por agentes fúngicos, displasia fibromuscular, gravidez, doenças do tecido conjuntivo e inflamações periarteriais que acompanham casos de colecistite e pancreatite. Entretanto, em mais da metade dos casos, nenhuma causa definida pode ser encontrada. Foi o que ocorreu em nosso caso, que não apresentava nenhum dos fatores anteriormente citados.

Os homens na faixa etária entre 45 e 85 anos são os mais frequentemente acometidos pelas dissecções das artérias esplâncnicas, correspondendo a $88 \%$ da série descrita por Sparks et al. ${ }^{7}$ assim como na série de Takayama et al. ${ }^{10}$.

Como em nosso paciente, aproximadamente a metade de todas as dissecções de artérias viscerais são assintomáticas $^{2,3,11}$, sendo achado de exames complementares. Nos casos de dissecção sintomática do tronco celíaco, Matsuo et al. ${ }^{3}$ relataram a possibilidade de icterícia quando há comprometimento da artéria hepática ou de pancreatite quando das arcadas pancreáticas associadas ao sintoma mais proeminente, que é a dor abdominal. Devido à rica circulação colateral proveniente da artéria mesentérica superior, as complicações trombóticas da dissecção do tronco celíaco são frequentemente bem toleradas.

Dentre os métodos complementares de diagnóstico a angiotomografia é considerado o método de escolha. A imagem mais frequentemente encontrada nesse exame é a presença de um flape de íntima, o que firma o diagnóstico de dissecção.

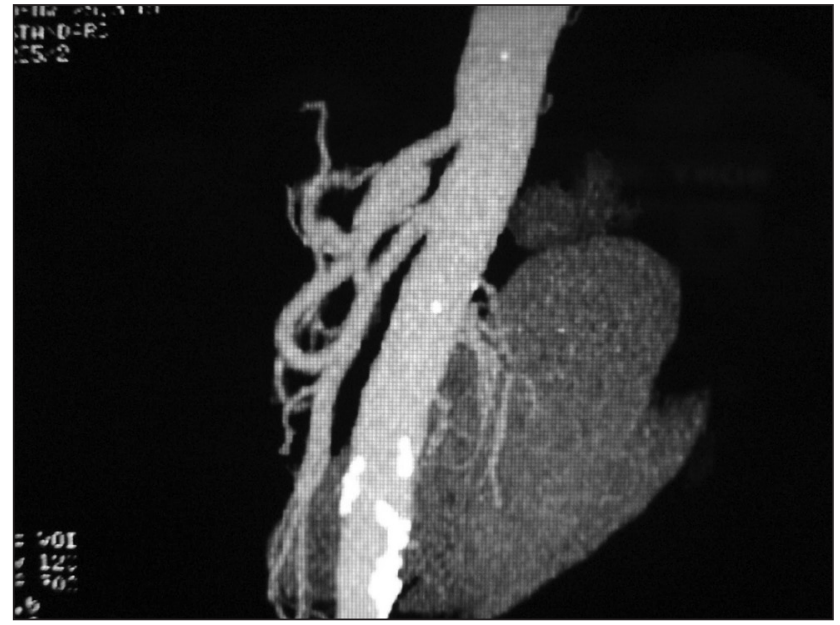

Figura 5 - Angiotomografia: ausência de dissecção aórtica

Outros achados são: presença de hematoma na parede arterial, que geralmente corresponde ao falso lúmen trombosado, formação de aneurisma e presença de um infiltrado no tecido gorduroso que circunda o tronco celíaco. Este último achado, às vezes encontrado na tomografia simples para investigação de dor abdominal, pode ser indicativo de uma nova tomografia realizada com contraste. D'Ambrosio et al. ${ }^{8}$ relatam inclusive ser esse um sinal prognóstico, que, quando presente, pode sugerir a extensão da dissecção para os vasos adjacentes. A angiotomografia permite ainda avaliar a extensão da dissecção, a formação de aneurisma e as suas medidas e o comprometimento de outras artérias.

O eco-Doppler é também uma modalidade diagnóstica de grande valia que pode ser facilmente realizada sem a exposição à irradiação, oferecendo dados precisos para o diagnóstico. Podem ser empregadas, ainda, a ressonância magnética e a angiografia.

A maioria dos autores sugere que as lesões assintomáticas não complicadas devem ser tratadas conservadoramente, apenas com monitoração do paciente com exames de imagem. A utilização de drogas antiplaquetárias ou anticoagulantes por 3 a 6 meses para evitar complicações tromboembólicas, assim como a modificação dos fatores de risco cardíaco para limitar a propagação da dissecção e reduzir o risco de ruptura, têm sido sugeridas. Em nosso paciente não utilizamos nenhuma medicação e ele apresenta-se assintomático há 25 meses.

O tratamento cirúrgico ou endovascular deverá ser realizado apenas nos casos em que a formação aneurismática tiver crescimento progressivo, sendo indicada a correção nos casos com aneurisma fusiforme com diâmetro transverso superior a 20 ou $24 \mathrm{~mm}$, o que corresponde a um diâmetro de 2,5 a 3,0 vezes superior ao diâmetro da artéria normal. 
A intervenção também estará indicada nos casos em que ocorrerem complicações tais como ruptura arterial, trombose da luz verdadeira com isquemia hepática e lesões múltiplas comprometendo o trato digestivo inferior.

Entretanto, em revisão que fizemos da literatura, pudemos observar que o tratamento conservador pode ser proposto com segurança para os pacientes com dissecção limitada e que no acompanhamento seriado com exames de imagem não tenham demonstrado evidências de ruptura ou expansão.

\section{Referências}

1. Woolard JD, Ammar AD. Spontaneous dissection of celiac artery: A case report. J Vasc Surg. 2007;45:1256-8.

2. Chaillou P, Moussu P, Noel SF, et al. Spontaneous dissection of celiac artery. Ann Vasc Surg. 1997;11:413-5.

3. Matsuo R, Ohta Y, Ohya Y, et al. Isolated dissection of celiac artery: a case report. Angiology. 2000;51:603-7.

4. Muller MF, Kim D. Spontaneous dissection of the hepatic artery. Abdom Imaging. 1995;20:462-5.

5. Yasuhara H, Shigematsu H, Muto T. Self-limited spontaneous dissection of the main trunk of the superior mesenteric artery. J Vasc Surg. 1998;27:776-9.

6. Suzuki S, Furui S, Kohtake H, et al. Isolated dissection of the superior mesenteric artery: CT findings in six cases. Abdom Imaging. 2004:29:153-7.
7. Sparks SR, Vasques JC, ergan JJ, Owens EL. Failure of nonoperative management of isolated superior mesenteric artery dissection. Ann Vasc Surg. 2000;14:105-9.

8. D’Ambrosio N, Friedman B, Siegel D, Katz D, Newatia A, Hines J. Spontaneous isolated dissection of the celiac artery: CT findings in adults. AJR Am J Roentgenol. 2007;188:W506-11.

9. Glehen O, Feugier P, Aleksic T, Delannoy P, Chealier JM. Spontaneous dissection of the celiac artery. Ann Vasc Surg. 2001;15:687-92.

10. Takayama T, Miyata T, Shirakawa M, Nagawa H. Isolated spontaneous dissection of the splanchnic arteries. J Vasc Surg. 2008;48:329-33.

11. Foord AG, Lewis RD. Primary dissection aneurysm of peripheral and pulmonary arteries: dissecting hemorrhage of media. Arch Pathol. 1959;68:553-77.

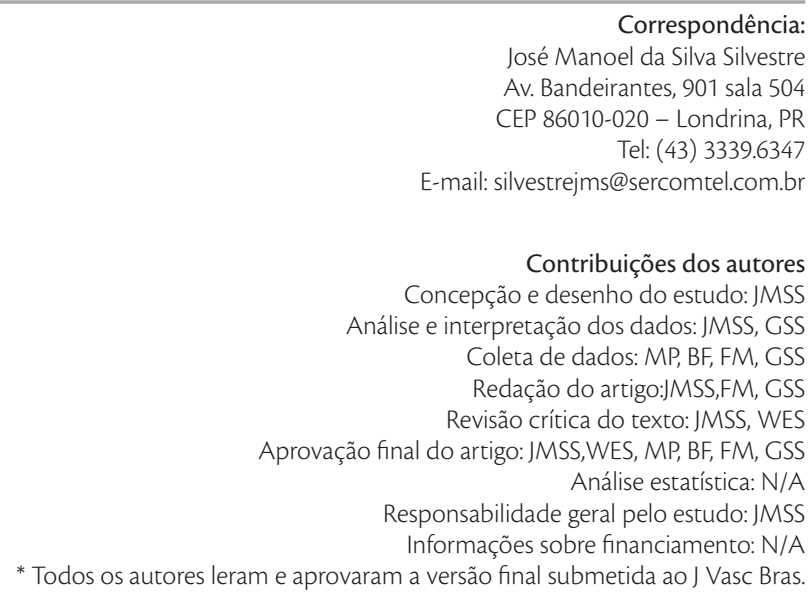

